\title{
What are the left-handed media and what is interesting about them?*
}

\author{
K. Yu. Bliokh ${ }^{\dagger}$ and Yu. P. Bliokh
}

\begin{abstract}
We review the intensively discussed ideas about wave propagation and refraction in media where both electric permittivity and magnetic permeability are negative. The criticism against negative refraction as violating the causality principle is considered. Starting from the initial wave equations, refraction of beams at the boundary of a left-handed medium is analyzed. The physics of a perfect lens formed by a flat layer of a left-handed material is considered.
\end{abstract}

\section{Contents}

\begin{tabular}{lll}
\hline & Introduction & 2
\end{tabular}

2 Negative-index media 2

3 Wave propagation and refraction in left-handed media 4

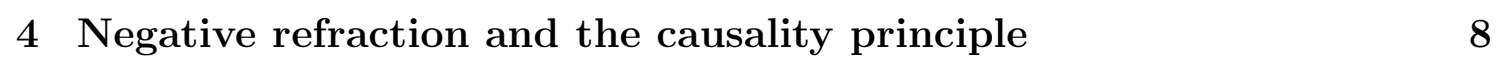

5 Wave and geometric optics of a left-handed medium 9

6 A perfect lens $\quad 14$

\begin{tabular}{lll}
\hline Conclusion & 19
\end{tabular}

*Published as Methodological Notes in Physics - Uspekhi 47 (4) 393 (2004).

${ }^{\dagger}$ Radio Astronomy Institute, National Academy of Scienses of Ukraine, 61002 Kharkov, Ukraine, e-mail: k_bliokh@mail.ru

‡Departmant of Physics, Technion, 32000 Haifa, Israel, e-mail: bliokh@physics.technion.ac.il 


\section{Introduction}

The term "left-handed media" was first introduced by V. G. Veselago in 1967 [1] for media with a negative refractive index ${ }^{1}$. The increase in the number of publications on this subject is clearly illustrated by the table (Fig. 1) published by J. B. Pendry in April 2003 in the introduction to a special issue of Optical Express [3]. A flood of interest in this problem at the end of the 1990s was initiated by the creation of composite media with negative refraction and their first experimental studies. What is so interesting about these media that has made many physicists abandon their current activities and turn to investigating them? The present paper is an attempt to answer this question.

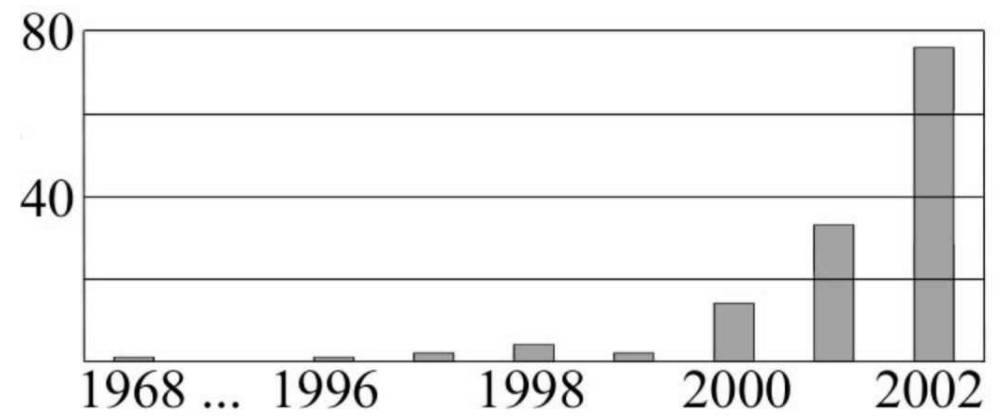

Figure 1: The number of publications on negative-index media (NIM) (from Ref. [3] )

\section{Negative-index media}

The sign of the refractive index of a medium depends on whether the phase and group velocities of a wave are parallel or antiparallel in this medium. In the first case, the group velocity is considered positive and in the second case it is considered negative. We believe that it is worth quoting L. I. Mandel'shtam's paper of 1945 [4] in this connection:

"Perhaps it should be stressed that the sign of the group velocity has a considerable effect on some phenomena that are usually discussed without even mentioning the group velocity.

I mean, for instance, reflection and refraction of a plane wave at the plane boundary between two nonabsorbing media.

\footnotetext{
${ }^{1}$ The concept of a negative refractive index was also first introduced by Veselago [2]
} 
In deriving the corresponding equations - for the direction of the refracted beam and for the amplitudes of the reflected and refracted waves - one makes an assumption, usually not mentioned explicitly, that the phase velocity of the refracted wave forms an acute angle with the normal to the boundary directed towards the "second" medium.

At the same time, according to the physical essence of the problem, this assumption should relate to the group velocity (to the velocity of energy propagation). The resulting equations are correct only because in real situations, as mentioned above, we deal with positive group velocities.

If the group velocity is negative, the requirement that the energy propagates from the boundary is equivalent to the requirement that the phase on the boundary is increasing. In this case, the refracted beam is directed not in a usual way but symmetrically with respect to the normal to the boundary."

In the same paper, Mandel'shtam notes that a spatially periodic medium (in his analysis, a crystal lattice) provides an example of a medium where the refractive index can be negative within a certain frequency range. Composite left-handed media, which were developed at the end of the nineties [5, 6] and caused an explosion of interest in this problem, are also spatially periodic. Such media have a negative refractive index for microwaves with frequencies of the order of $10 \mathrm{GHz}$.

Periodic waveguide systems, or slow wave structures, have been well known in microwave electronics for a long time. Waves with negative refractive index are also well known: these are backward waves, or negative-dispersion waves. So what is the difference between the "old" slow wave structures and the new ones, the left-handed media? A principal difference is that the traditional "old" slow wave structures are one-dimensional, while the left-handed media are multi-dimensional (two- or three-dimensional). Refraction of a wave at the interface of two media is a multi-dimensional effect and is therefore absent in slow wave structures.

Investigation of multi-dimensional periodic structures was started long ago (see Refs. [7, 8, 9] and the references therein) but the authors of Refs. [5, 6] do not cite any previous works. This is possibly because they are unaware of papers published in Russian; another reason may be that their approach to the problem is totally new. The authors of theoretical paper [5] consider the possibility of creating a periodic structure with a surface where low-frequency weakly decaying waves could exist, similarly to plasmons - collective waves in metals. This is possible in a medium with negative electric permittivity (dielectric constant). The authors of Ref. [5] have shown that a periodic grating of thin wires has low losses and negative electric permittivity $\varepsilon<0$ for electromagnetic waves with frequencies of $1-10 \mathrm{GHz}$. Later, it was shown [6] that a periodic grating of ring cavities has negative magnetic permeability $\mu<0$ in the same range. By combining both gratings, a left-handed 
negative-index medium was formed [10, 11].

It should be said to the credit of Western scientists that they managed to make their research a tremendous success due to publications in respected physics journals as well as in popular publications, including newspapers ${ }^{2}$. But we now put the questions of prestige aside and turn to physics.

As another example [12] of a left-handed medium, we mention photonic crystals, which are media with a spatially periodic refractive index $n(\mathbf{r})$.

Thus, left-handed media are two- or three-dimensional periodic structures. In such anisotropic media, the angle between the phase and group velocities of a wave can be different. The only exceptions are spatially periodic media that are isotropic $^{3}$ in the long-wave approximation, when the wavelength $\lambda$ is much larger than the period $d, \lambda \gg d$. In this case, a medium can be characterized by effective electric permittivity $\varepsilon$ and effective magnetic permeability $\mu$, and waves can be classified as forward and backward. It was shown in Ref. [12 that in photonic crystals, where modulation of parameters is rather strong, anisotropy in some frequency ranges near bandgaps can be negligibly small.

\section{Wave propagation and refraction in left-handed media}

We consider a plane electromagnetic wave propagating in a medium with a scalar electric permittivity $\varepsilon$ and a magnetic permeability $\mu$. If $\varepsilon>0$ and $\mu>0$, the electric field $\vec{E}$, magnetic field $\vec{H}$, and wavevector $\vec{k}$ form a right-handed vector triplet; if $\varepsilon<0$ and $\mu<0$, they form a left-handed triplet. This is the origin of classifying media into left-handed and right-handed [1. The energy flux of a wave is given by the Poynting vector

$$
\vec{S}=\frac{c}{4 \pi}[\vec{E} \vec{H}],
$$

which always forms a right-handed triplet with the vectors $\vec{E}$ and $\vec{H}$. Therefore, the group and phase velocities are parallel in right-handed media (positive group velocity) and are anti-parallel in left-handed media (negative group velocity).

Refraction of a plane wave at the interface of a left-handed medium and a righthanded one looks quite unusual. For definiteness, we consider a wave propagating

\footnotetext{
${ }^{2} \mathrm{~A}$ large and constantly updated collection of references can be found at http://physics.ucsd.edu/ drs/left_home.htm

${ }^{3}$ While we were preparing this material for publication, a paper appeared [13] where refraction of light at the interface of two anisotropic media with different directions of optic axes was erroneously interpreted as a manifestation of the negative refractive index (for more details, see Ref. 14])
} 
from a usual right-handed medium (which is assumed to be the vacuum in what follows) to the plane boundary of a left-handed medium. The group velocity of the refracted wave is directed from the boundary and its phase velocity is directed towards the boundary. This means that the phase velocities of both waves are directed towards the boundary, and hence the phases of both waves increase on the boundary. This is possible only if the propagation directions of both waves (group velocities) are on the same side of the normal to the boundary (Fig. 21). In other words, the refractive index $n$ entering Snell's law for the left-handed medium is negative, $n=-\sqrt{\varepsilon \mu}$.

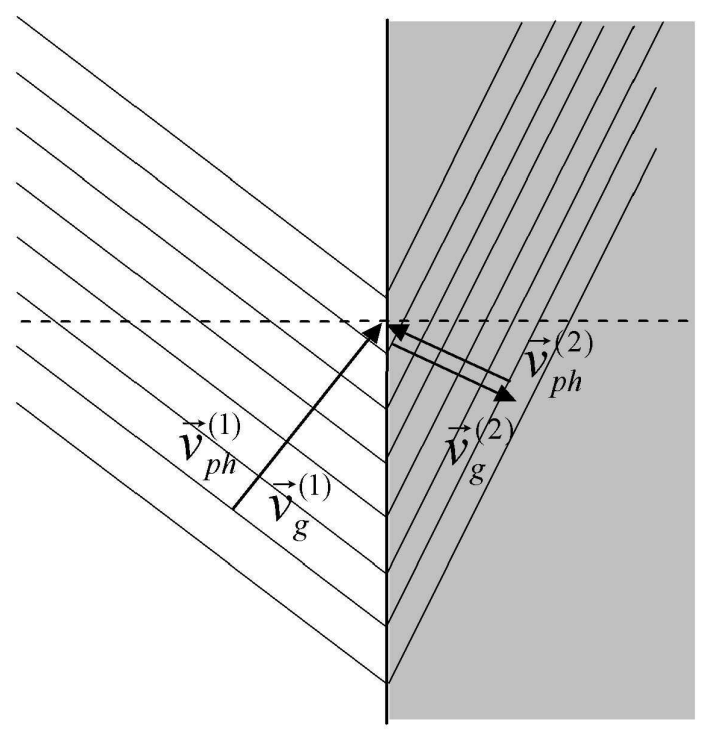

Figure 2: Refraction of a plane wave.

The fact that the incident and refracted waves are on the same side of the normal to the boundary enables one to manufacture quite unusual optic elements of lefthanded media. For instance, a plane-parallel plate made of a left- handed material works as a collecting lens, as can be readily seen from the ray diagram in Fig. [3. Such a lens has a remarkable feature: it has no focal plane. It therefore forms a three-dimensional image of an object, which makes it similar to a mirror. But in contrast to a mirror, it forms a real image, and this feature opens new possibilities for three-dimensional photography. Of course, this flat lens also has a certain drawback: for an object to be imaged, it must be placed sufficiently close to the surface of the lens. For instance, for an object placed near a lens made of a "perfect" left-handed material $(\varepsilon=\mu=-1)$, only those points whose distance from the lens surface does 
not exceed the thickness of the lens have real images (Fig. (4).

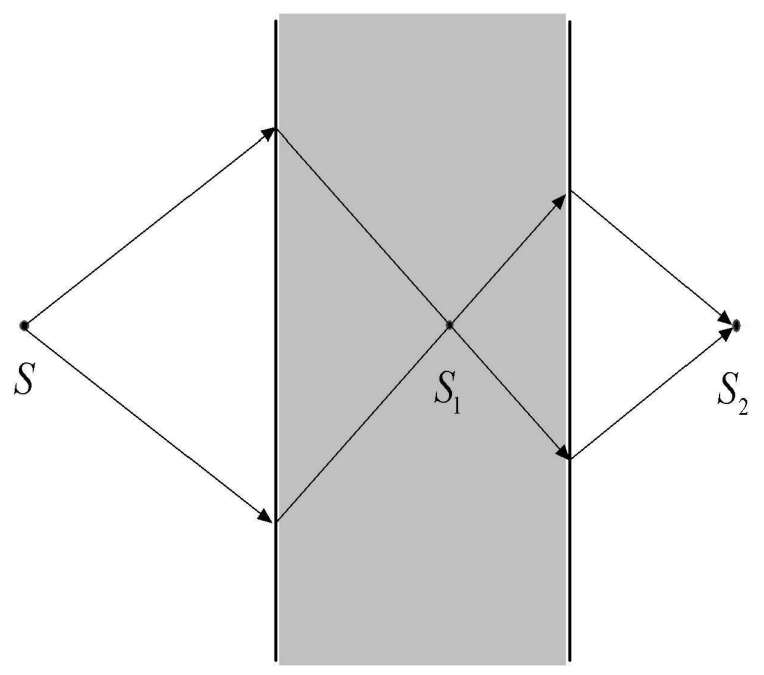

Figure 3: A plane-parallel plate of a left-handed material acts as a collecting lens.

It is no accident that we have used the term "perfect left-handed medium" for the medium with $\varepsilon=\mu=-1$. Indeed, such a medium has some additional interesting features. First, a perfect left-handed medium has zero reflection coefficient: all the energy of the incident wave is passed to the refracted wave. Second, a plane-parallel plate of a perfect left-handed material forms a perfect image because the phase incursion along any trajectory between the object and the image equals zero. This can be easily understood by noting that for any beam traveling from the object to the image, half of the path is in the ordinary medium and the other half is in the perfect left-handed medium. Because phase velocities in the two media have equal values but opposite directions, phase delays along the two parts of any trajectory exactly compensate each other.

But these are not all the wonderful properties of a perfect left-handed medium. In 2000, Pendry published a paper "Negative refraction makes a perfect lens" [15]. Here, "a perfect lens" means a lens whose resolving power exceeds the limit set by the wave nature of light. In order to understand the author's reasoning, we consider a monochromatic light source placed in the plane parallel to a plate of a perfect left-handed material. Let the distribution of the field (magnetic field, for instance) in the source plane be given by a function $H_{0}(x)$. This function can be represented 


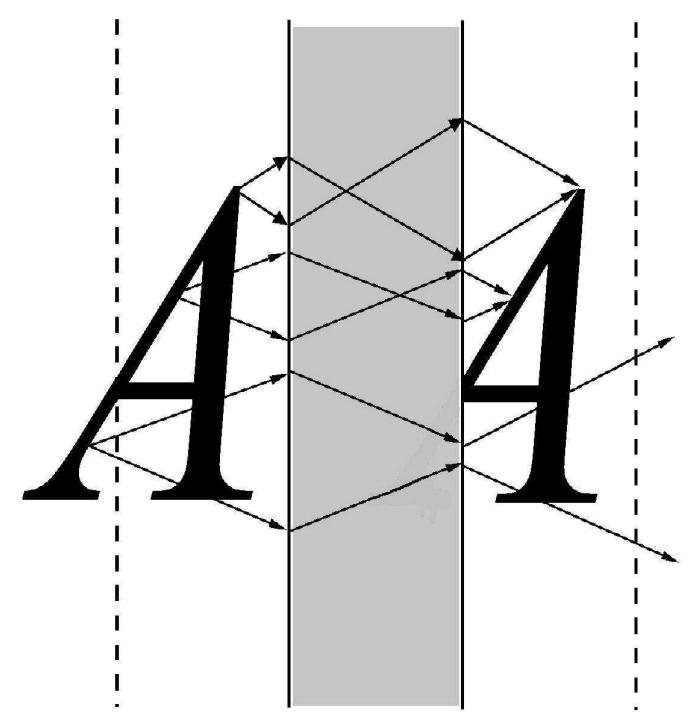

Figure 4: Athree-dimensional image obtained by means of a plane-parallel plate made of a left-handed material.

as a spatial Fourier transform,

$$
H_{0}(x)=\int_{-\infty}^{+\infty} H_{k}\left(k_{\perp}\right) \exp \left(i k_{\perp} x\right) d k_{\perp}
$$

The field of the wave propagating along the $z$ axis from the source to the plate can be represented as

$$
H(x, z)=\int_{-\infty}^{+\infty} H_{k}\left(k_{\perp}\right) \exp \left(i k_{\perp} x\right) \exp \left(i \sqrt{\omega^{2} / c^{2}-k_{\perp}^{2}} z\right) d k_{\perp}
$$

It can be seen from representation (2) that the Fourier components with $k_{\perp}>$ $\omega / c$ decay exponentially with the distance from the source. These nonpropagating waves are usually neglected. Omitting harmonics with $k_{\perp}>\omega / c$ means losing the information about the details of the image that are smaller than the wavelength $\lambda=2 \pi c / \omega$.

In Ref. [15], it is noted that the exponential decay of nonpropagating waves affects only their amplitudes but not their phases. Therefore, one can completely restore the information about the spatial structure of the source by amplifying these waves. It is shown in Ref. [15] that a plate of a perfect left-handed material can 
serve as this "perfect" amplifier, increasing the amplitudes of nonpropagating waves by exactly the same value as that of their decay.

These unusual (and quite tempting from the practical standpoint) properties of left-handed materials have caused an outburst of publications during the recent years. However, not all the participants in the discussion in the literature agree with the optimistic conclusions that we have briefly reviewed in this section.

\section{Negative refraction and the causality principle}

In 2002, Physical Review Letters published a paper entitled "Wave refraction in negative-index media: always positive and very inhomogeneous" [16]. The authors claim that negative refraction contradicts the causality principle and therefore does not exist. The authors' reasoning can be most easily understood with the help of Fig. 5 ,

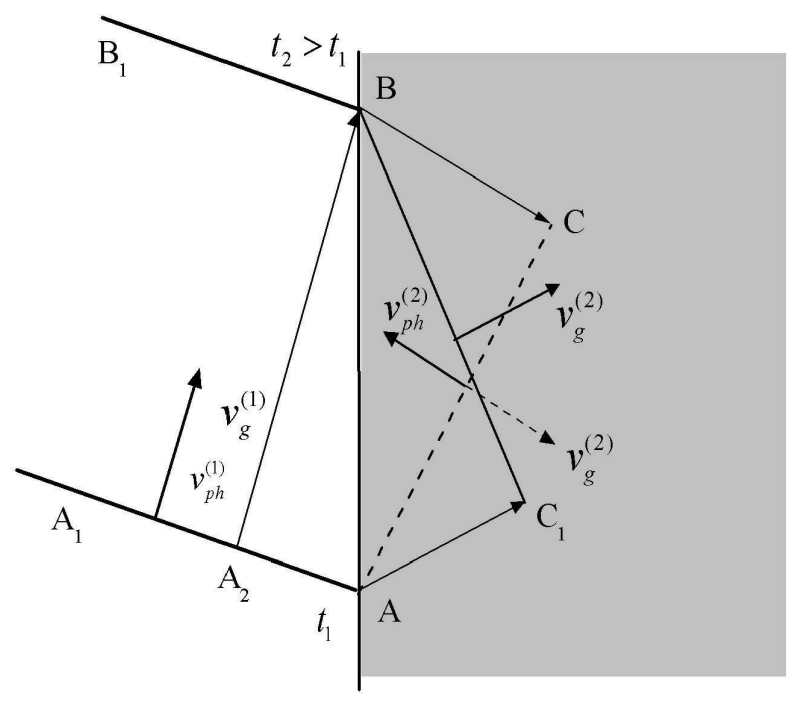

Figure 5: Negative refraction and the causality principle.

Let a wave packet be incident on an interface of the vacuum (left) and a lefthanded isotropic medium (right). The group velocity $\vec{v}_{g}^{(1)}$ and the phase velocity $\vec{v}_{p h}^{(1)}$ of the wave packet in the vacuum coincide. Let the wave packet front coincide with the line $A A_{1}$ at time $t_{1}$ and with the line $B B_{1}$ at time $t_{2}>t_{1}$. In the lefthanded medium, the phase velocity of the refracted wave $\vec{v}_{p h}^{(2)}$ is directed towards 
the boundary. According to the authors' reasoning, if the group velocity $\vec{v}_{g}^{(2)}$ of the refracted wave were directed oppositely to the phase velocity, then the front of the refracted wave packet would be oriented along the line $A C$ (dashed line in Fig. 5), orthogonally to the group velocity direction. Because the time required for the front displacement along the path $A A_{1} \rightarrow B B_{1} \rightarrow A C$ is the same for all points of the front, it follows from Fig. 5 that the signal should travel along the path $A_{2} B C$ with infinite velocity. This violates the causality principle. The authors conclude that the front of the refracted wave should coincide with the bold line $B C_{1}$ and the group velocity should be orthogonal to it. They suggest that one should distinguish between phase and group refractive indices. The first describes the change in the direction of the phase velocity, and it is indeed negative for left-handed media. But the group refractive index describes the direction of energy propagation, and it is positive. Because the beam diagram used in geometric optics (like the one shown in Fig. 3) should indicate the direction of energy propagation and not phase propagation, there can be no focusing properties of the plate, to say nothing of forming a "perfect" image.

Further, a large difference between the group and phase velocities of the refracted wave means strong dispersion; therefore, a wave packet "spreads" after passing the interface of the two media, its amplitude decreases very fast, and decay of the incident wave occurs instead of focusing.

The standpoint of the authors of Ref. [16] is described in full detail, with pictures and animations, at http://www.utexas.edu/research/cemd/nim/

\section{Wave and geometric optics of a left-handed me- dium}

Two flaws can be seen in the above proof that the "actual" (i.e., group) refraction cannot be negative. First, the authors claim that group and phase velocities can be noncollinear in an isotropic medium. Second, to investigate the focusing properties of an optical system (in our case, a plane-parallel plate) one does not need to consider a multi-frequency wave packet, hence, dispersion of the medium is not relevant.

In order to find the source of error in Ref. [16], we consider refraction of a narrow monochromatic light beam in the framework of the wave equation. First of all, this approach enables us to do without the concept of group velocity direction as the direction of energy propagation. (Indeed, the beam direction is evidently the same as the direction of energy propagation.) Second, using the wave equation approach allows finding whether the geometric optics approximation is applicable to left-handed media. 
Let the incident wave be the exact solution of the two-dimensional Maxwell equations in the vacuum. It can be represented as the Fourier integral

$$
\begin{array}{r}
E_{x}=\frac{1}{\pi} \int_{-\infty}^{\infty} d k_{\perp} \frac{\sin \left(k_{\perp} a\right)}{k_{\perp}} \frac{\sqrt{k_{0}^{2}-k_{\perp}^{2}}}{k_{0}} \exp \left(i k_{\perp} x+i \sqrt{k_{0}^{2}-k_{\perp}^{2}} z\right), \\
E_{z}=-\frac{1}{\pi} \int_{-\infty}^{\infty} d k_{\perp} \frac{\sin \left(k_{\perp} a\right)}{k_{0}} \exp \left(i k_{\perp} x+i \sqrt{k_{0}^{2}-k_{\perp}^{2}} z\right), \\
H_{y}=\frac{1}{\pi} \int_{-\infty}^{\infty} d k_{\perp} \frac{\sin \left(k_{\perp} a\right)}{k_{\perp}} \exp \left(i k_{\perp} x+i \sqrt{k_{0}^{2}-k_{\perp}^{2}} z\right) .
\end{array}
$$

Here, $k_{0}=\omega / c$. We consider the two-dimensional case for simplicity. Electric and magnetic fields in (3) form a monochromatic wave (we have omitted the factor $e^{-i \omega t}$ in all terms). The source of this wave is placed in the plane $z=0$ and has the following distribution of magnetic field ${ }^{4}$ :

$$
\begin{array}{r}
H_{0}(x) \equiv H_{y}(x, 0)=1 \text { at }|x|<a \\
H_{0}(x)=0 \text { at }|x|>a .
\end{array}
$$

Here, $a$ denotes the size of the source in the $x$ direction. Such a wave forms a beam propagating along the $z$ axis. The angular divergence of the beam, $\alpha \sim\left(k_{0} a\right)^{-1}$, is small if the wavelength is small compared to the source size, $k_{0} a \gg 1$. In what follows, we assume this condition to be satisfied.

The field (3) at an arbitrary point $(x, z)$ consists of propagating $\left(\left|k_{\perp}\right|<k_{0}\right)$ and decaying $\left(\left|k_{\perp}\right|>k_{0}\right)$ plane waves. At a large distance from the source, decaying waves can be neglected (we discuss below whether they can be amplified in a lefthanded medium), and the integrals in (3) must be calculated for $-k_{0}<k_{\perp}<k_{0}$. To simplify the notation, we omit the integration limits in what follows.

Let the plane boundary between the vacuum and the medium intersect the $z$ axis at a point $z=z_{0}$ and the normal to the surface form the angle $\varphi$ with the $z$ axis (Fig. 6). We introduce the new coordinates $x^{\prime}, z^{\prime}$ with the origin at the point $\left(0, z_{0}\right)$. The $x^{\prime}$ axis is directed along the boundary and the $z^{\prime}$ axis along the inner normal. At the boundary $z^{\prime}=0$, the magnetic field of the incident wave can be written as

$$
\begin{gathered}
H_{y}^{(i n)}=\frac{1}{\pi} \int d k_{\perp}^{\prime} \frac{\sqrt{k_{0}^{2}-k_{\perp}^{2}\left(k_{\perp}^{\prime}\right)}}{\sqrt{k_{0}^{2}-k_{\perp}^{\prime 2}}} \frac{\sin \left[k_{\perp}\left(k_{\perp}^{\prime}\right) a\right]}{k_{\perp}\left(k_{\perp}^{\prime}\right)} \exp \left[i k_{\perp}^{\prime} x^{\prime}+i z_{0} \sqrt{k_{0}^{2}-k_{\perp}{ }^{2}\left(k_{\perp}^{\prime}\right)}\right], \\
k_{\perp}\left(k_{\perp}^{\prime}\right)=k_{\perp}^{\prime} \cos \varphi-\sin \varphi \sqrt{k_{0}^{2}-{k_{\perp}^{\prime}}^{2}} .
\end{gathered}
$$

\footnotetext{
${ }^{4}$ This boundary condition was used by Kirchhoff in solving the problem of light diffraction by a slit.
} 
Fourier components of the electric field can be expressed in terms of the magnetic field components, and we do not therefore consider them here explicitly.

Using the boundary conditions

$$
\begin{gathered}
E_{t}^{(i n)}\left(k_{\perp}^{\prime}\right)+E_{t}^{(r e f)}\left(k_{\perp}^{\prime}\right)=E_{t}^{(t r)}\left(k_{\perp}^{\prime}\right), \\
H_{t}^{(i n)}\left(k_{\perp}^{\prime}\right)+H_{t}^{(r e f)}\left(k_{\perp}^{\prime}\right)=H_{t}^{(t r)}\left(k_{\perp}^{\prime}\right)
\end{gathered}
$$

for the Fourier components of the tangential and normal parts of the fields, we can calculate the amplitudes of the Fourier components of the reflected (labeled by "ref") and the refracted (labeled by "tr") waves on the surface and, hence, at any space point $x^{\prime}, z^{\prime}$. This procedure is standard, and the only peculiar feature in the case of a left-handed medium is the unusual sign of the normal part

$$
k_{\|}^{\prime}= \pm \sqrt{k_{0}^{2} \varepsilon \mu-k_{\perp}^{2}}
$$

of the refracted wave wavevector. As we have mentioned above, the sign is chosen such that the energy flux in the refracted wave is directed from the boundary. In a left-handed medium, the wavevector, the magnetic field, and the electric field form a left-handed triplet, and hence $k_{\|}^{\prime}<0$.

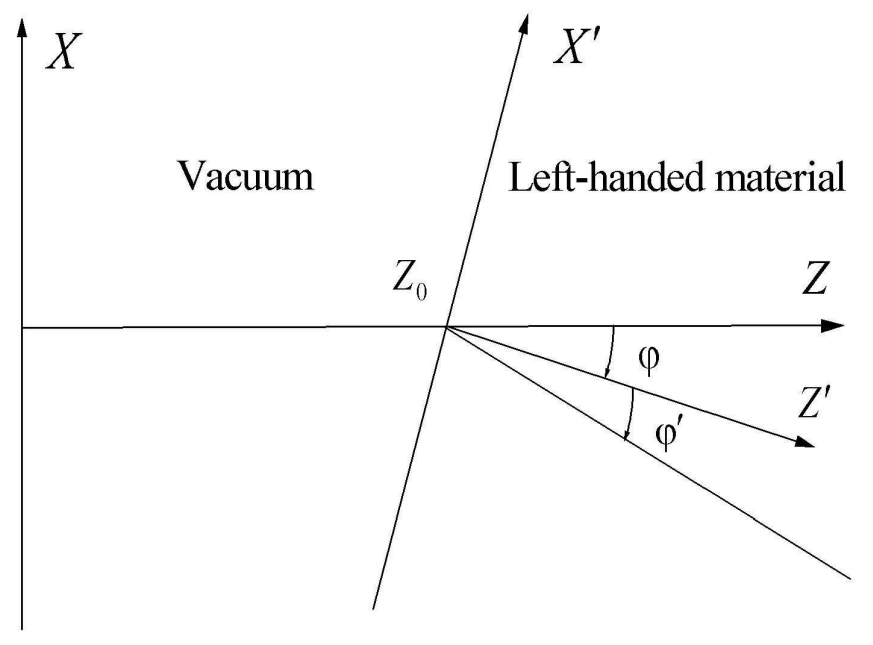

Figure 6:

Taking into account that at an arbitrary point of the medium $x^{\prime}, z^{\prime}$, each Fourier component differs from its value at the boundary by the factor

$$
\exp \left(i \operatorname{sgn} \varepsilon \sqrt{\mathrm{k}_{0}^{2} \varepsilon \mu-\mathrm{k}_{\perp}^{\prime 2} \mathrm{z}^{\prime}}\right)
$$


we easily express the magnetic field of the refracted wave as

$$
H_{t}^{(t r)}=\frac{1}{\pi} \int d k_{\perp}^{\prime} f\left(k_{\perp}^{\prime}\right) \frac{\sin \left[k_{\perp}\left(k_{\perp}^{\prime}\right) a\right]}{k_{\perp}\left(k_{\perp}^{\prime}\right)} \exp \left[i \Phi\left(k_{\perp}^{\prime}\right)\right],
$$

where

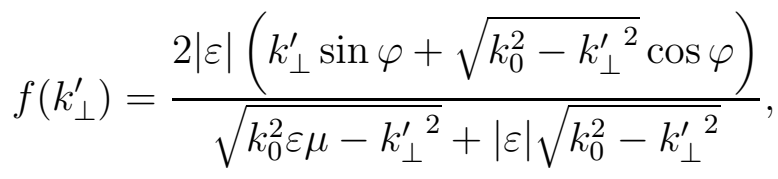

$$
\begin{aligned}
& \Phi\left(k_{\perp}^{\prime}\right)=z_{0}\left(k_{\perp}^{\prime} \sin \varphi+\sqrt{k_{0}^{2}-{k_{\perp}^{\prime}}^{2}} \cos \varphi\right)+ \\
& k_{\perp}^{\prime} x^{\prime}+\operatorname{sgn} \varepsilon \sqrt{k_{0}^{2} \varepsilon \mu-k_{\perp}^{\prime 2}} z^{\prime} .
\end{aligned}
$$

Expression (17) is valid for both right-handed and left-handed media. The only difference is the factor $\operatorname{sgn} \varepsilon$ in the phase $\Phi\left(k_{\perp}^{\prime}\right)$.

We now analyze Eq (17). In the simplest case of a perfect left-handed medium $(\varepsilon=\mu=-1)$ with $\varphi=0$,

$$
H_{t}^{(t r)}\left(x, z^{\prime}\right)=H_{y}\left(x, z_{0}-z^{\prime}\right)
$$

This relation implies that after crossing the boundary, a divergent beam becomes convergent and restores the image of the object in the plane $z=2 z_{0}$. In the general case, the amplitude of the magnetic field is maximal when the stationary point $k_{s}$, which is determined by the condition $d \Phi /\left.d k_{\perp}^{\prime}\right|_{k_{\perp}^{\prime}=k_{s}}=0$, coincides with the point $k_{\perp}\left(k_{\perp}^{\prime}\right)=0$ where the function $\sin \left(k_{\perp} a\right) / k_{\perp}$ is maximal:

$$
k_{\perp}\left(k_{s}\right)=0 .
$$

This condition is satisfied on the line

$$
\frac{x^{\prime}}{z^{\prime}} \equiv \tan \varphi^{\prime}=\frac{\operatorname{sgn} \varepsilon \sin \varphi}{\sqrt{\varepsilon \mu-\sin ^{2} \varphi}},
$$

which is natural to interpret as the central line of the refracted beam. The amplitude of the wave and, hence, the energy flux are maximal along this direction; therefore, the group velocity is directed along it. It is easy to see that the angles $\varphi$ and $\varphi^{\prime}$ are related through Snell's law,

$$
\frac{\sin \varphi}{\sin \varphi^{\prime}}=\operatorname{sgn} \varepsilon \sqrt{\varepsilon \mu}
$$


where the sign of $\varepsilon$ indicates whether the incident and refracted beams are on the same side of the normal to the boundary. We also note that

$$
\tan \varphi^{\prime}=\frac{k_{\perp}^{\prime}}{k_{\|}^{\prime}}=\frac{\operatorname{sgn} \varepsilon \mathrm{k}_{\perp}^{\prime}}{\sqrt{\varepsilon \mu \mathrm{k}_{0}^{2}-\mathrm{k}_{\perp}^{\prime 2}}}
$$

and therefore the refraction law is the same for the phase and group velocities.

Thus, by exactly solving the Maxwell equations, we see that there is no difference between phase and group refractive indices and that the refractive index is negative for a left-handed medium.

We next consider the contradiction between the exact solution of the Maxwell equations and the statement in Ref. [16 that "causality and finite signal speed preclude negative refraction for any waves incident on any material, including NIM" (negative-index medium). We return to the scheme of wave packet refraction shown in Fig. 66 this time, however, negative refraction is assumed. Figure 7 shows the positions of the wave packet at three consecutive time instants, $t_{1}<t_{2}<t_{3}$, which allows reconstructing the shape of the packet in the medium. Indeed, the front of the refracted packet coincides with the line $B C_{1}$ shown in Fig. 15, but the statement in Ref. [16] that the normal to the front gives the group velocity direction is erroneous. Hence, the conclusion that the phase and group velocities in a left-handed medium are noncollinear is also erroneous. Actually, when the packet crosses the boundary of a left-handed medium, its shape changes: an initially "straight" packet becomes a "slanting" one. (In Ref. [17], such behavior was called "crab-like motion".) Certainly, there is no causality violation here.

We had two reasons to choose the test solution of the Maxwell equations in the form of a narrow wave beam in this section. First, it allowed us to resolve the paradox in Ref. [16] without using the notion of group velocity; second, this way we could formally demonstrate that geometric optics (the concept of beams) is applicable to left-handed media. Actually, we never used the inequality $k_{0} a \gg 1$; therefore, the solutions we obtained are also valid for describing other situations.

We finally note that after paper [16] had been published, a number of papers appeared where the authors, by means of analytic [17, 18, or numerical [19, 20] methods, came to the same conclusion as the one we make in this section. However, in all those papers, nonmonochromatic waves were considered. In our opinion, this is not necessary for solving the present problem and can make the wrong impression that the methods of traditional optics, where nonmonochromaticity is considered only as a source of chromatic aberrations, cannot be applied to left-handed media. 


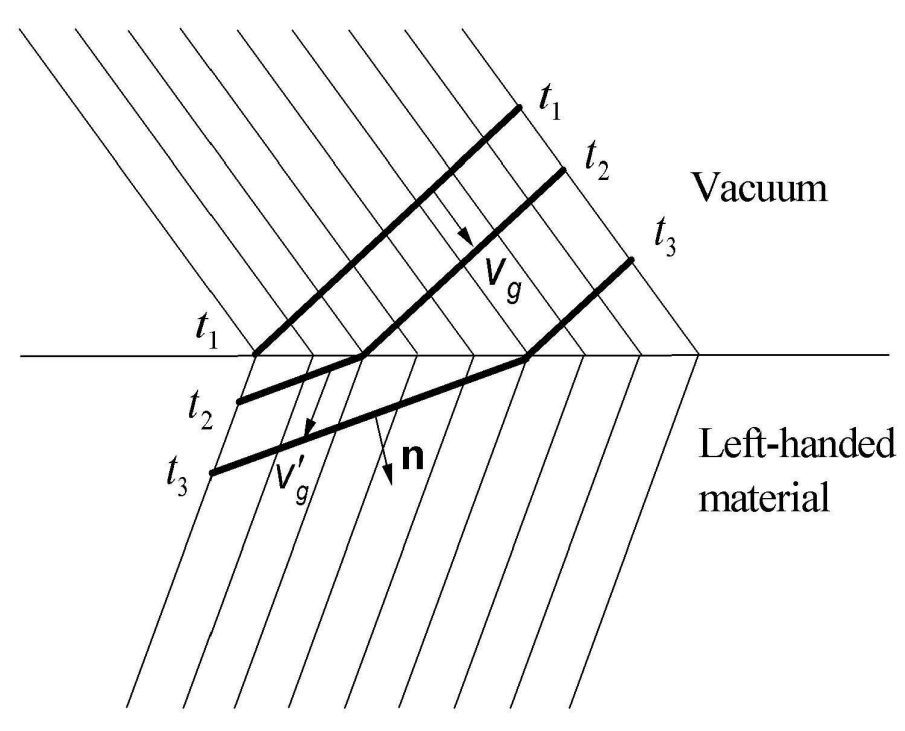

Figure 7: Transformation of a wave packet at the boundary of a left-handed medium.

\section{A perfect lens}

In analyzing equation (7), we have already considered the imaging of a source inside a left-handed medium in the simplest case of $\varepsilon=\mu=-1$ and a flat source placed parallel to the boundary of the medium. The same relation (17) can be used in a more general case where the source has arbitrary orientation relative to the boundary. We express $k_{\perp}^{\prime}$ through $k_{\perp}$ and write Eq.(17) in the form

$$
H_{t}^{(t r)}=\frac{1}{\pi} \int_{-\infty}^{\infty} d k_{\perp} \frac{\sin \left(k_{\perp} a\right)}{k_{\perp}} \exp \left[i \Phi\left(k_{\perp}\right)\right]
$$

where

$$
\Phi\left(k_{\perp}\right)=k_{\perp}\left(x^{\prime} \cos \varphi+z^{\prime} \sin \varphi\right)+\sqrt{k_{0}^{2}-k_{\perp}^{2}}\left(z_{0}+x^{\prime} \sin \varphi-z^{\prime} \cos \varphi\right) .
$$

The field given by (9) has the same form as the source field $H_{0}(x)$ on the line $z_{0}=z^{\prime} \cos \varphi+x^{\prime} \sin \varphi$ :

$$
H_{t}^{(t r)}\left(x^{\prime}, z^{\prime}=z_{0} / \cos \varphi+x^{\prime} \tan \varphi\right)=H_{0}\left(z_{0} \tan \varphi+x^{\prime} / \cos \varphi\right) .
$$

Relation (10) means that the source and its image are placed symmetrically with respect to the boundary. Similarly, one can obtain relations in a more general case of a medium with arbitrary $\varepsilon$ and $\mu$. Without presenting these relations, we only 
mention that the source field can be exactly restored only in the case of a perfect lefthanded medium. In all other cases, the image has imperfections. Monochromatic aberrations can be avoided if the lens is made of an anisotropic left-handed material with specially chosen dispersion dependence [21, but we do not consider anisotropic media in the framework of this paper. The properties of anisotropic left-handed media are considered in detail in Ref. 9].

We recall that the accuracy of restoring the image inside a plane layer of a perfect left-handed medium is limited by the wavelength, as in usual optical systems. But this is true only in the case where the left-handed medium does not amplify nonpropagating waves with $k_{\perp}>k_{0}$. At first sight, the statement in Ref. 15] that a layer of a left-handed medium amplifies nonpropagating waves and hence enables one to exceed the wave limit for the accuracy of image restoration looks erroneous because a left-handed medium is a passive medium. But we refrain from hasty conclusions and try to scrutinize the problem. Because the problem is linear, we can restrict consideration to a single Fourier component of the field, $\sim \exp \left(i k_{\perp} x\right)$.

We start again from the problem of a plane wave incident on an interface of a right-handed medium and a left-handed one. Let the magnetic field $H_{y}$ of the incident wave be $H^{(i n 0)} \exp \left(i k_{z} z\right)$, the plane $z=z_{l}$ being the interface of the media.

The magnetic fields of the transmitted and reflected waves occurring at the interface are represented, respectively, as

$$
H^{(t r 1)} \exp \left(i q_{z} z\right)\left(z>z_{l}\right), \quad H^{(r e f 1)} \exp \left(-i k_{z} z\right)\left(z<z_{l}\right)
$$

where

$$
k_{z}=\sqrt{k_{0}^{2}-k_{\perp}^{2}}, \quad q_{z}=\sqrt{k_{0}^{2} \varepsilon \mu-k_{\perp}^{2}}
$$

are the respective longitudinal wavevector components in the right-handed medium and the left-handed medium.

Boundary conditions (6) provide the following relations between the amplitudes of the three waves:

$$
\begin{gathered}
H^{(i n 0)} e^{i k_{z} z_{l}}+H^{(r e f 1)} e^{-i k_{z} z_{l}}=H^{(t r 1)} e^{i q_{z} z_{l}} \\
k_{z} H^{(i n 0)} e^{i k_{z} z_{l}}-k_{z} H^{(r e f 1)} e^{-i k_{z} z_{l}}=\frac{q_{z}}{\varepsilon} H^{(t r 1)} e^{i q_{z} z_{l}}
\end{gathered}
$$

Equations (11) lead to the expressions for $H^{(r e f 1)}$ and $H^{(t r 1)}$ that solve the problem:

$$
\begin{gathered}
H^{(t r 1)}=\frac{2 \varepsilon k_{z}}{\varepsilon k_{z}+q_{z}} e^{i\left(k_{z}-q_{z}\right) z_{l}} H^{(i n 0)} \\
H^{(r e f 1)}=\frac{\varepsilon k_{z}-q_{z}}{\varepsilon k_{z}+q_{z}} e^{2 i k_{z} z_{l}} H^{(i n 0)} .
\end{gathered}
$$


We note the denominators in Eqs. (12). If both the incident wave and the transmitted wave are propagating ones, then the denominators are nonvanishing at any values of $k_{\perp}$, because $\operatorname{sgn} \varepsilon=\operatorname{sgn} q_{z}$. But if either the incident wave or the transmitted wave is a nonpropagating one, for which $k_{\perp}^{2}>k_{0}^{2}, k_{\perp}^{2}>k_{0}^{2} \mu \varepsilon$, then the denominators can vanish:

$$
\varepsilon k_{z}+q_{z} \equiv \varepsilon \sqrt{k_{0}^{2}-k_{\perp}^{2}}+\sqrt{k_{0}^{2} \varepsilon \mu-k_{\perp}^{2}}=0 .
$$

The signs in front of the roots must be chosen such that both the transmitted and the reflected waves decay with increasing the distance from the interface, i.e., such that Eq. (13) can be written as

$$
|\varepsilon| \sqrt{k_{\perp}^{2}-k_{0}^{2}}-\sqrt{k_{\perp}^{2}-k_{0}^{2} \varepsilon \mu}=0 .
$$

Equation (14) is the dispersion equation of surface eigenmodes, which can exist on the interface of a right-handed medium and a left-handed one in the absence of a source field $H^{(i n 0)} 22$.

If the wavevector $k_{\perp}$ of the source field coincides with the solution to dispersion equation (14), then the amplitudes of the transmitted and the reflected waves become infinite, because the external field acts as a resonant force exciting nondecaying eigenmodes of the medium. Hence, the interface itself is a resonant "amplifier" of nondecaying waves. Similarly to the case of a usual cavity, taking nonzero losses in the medium into account restricts the amplitude of the resonant surface wave and determines the resonance linewidth.

Before passing to the plane-parallel lens, we note an interesting feature of surface waves: their energy flux is directed along the surface and changes sign on the surface. In the case of a perfect left-handed medium, the fluxes on the right and on the left of the surface have equal absolute values, and therefore the total energy flux along the surface is equal to zero.

We now consider a plane wave incident on a plane-parallel plate of a left-handed material. Let the second boundary of the plane coincide with the plane $z=z_{r}$. In addition to the previously considered waves, two new waves appear now: the one reflected from the second boundary and the one passing through the plate. Their magnetic fields are denoted by

$$
H^{(r e f 2)} \exp \left(-i q_{z} z\right), \quad H^{(t r 2)} \exp \left(i k_{z} z\right)
$$

respectively. From the boundary conditions on the two surfaces, taking all waves into account, we express their amplitudes as

$$
H^{(t r 1)}=\frac{1}{2 D\left(k_{\perp}\right)}\left(1+\frac{k_{z} \varepsilon}{q_{z}}\right) e^{-i q_{z} z_{r}+i k_{z} z_{l}},
$$




$$
\begin{array}{r}
H^{(r e f 1)}=-\frac{i}{2 D\left(k_{\perp}\right)}\left(\frac{k_{z} \varepsilon}{q_{z}}-\frac{q_{z}}{k_{z} \varepsilon}\right) \sin \left(q_{z} d\right) \\
H^{(t r 2)}=\frac{1}{D\left(k_{\perp}\right)} e^{-i k_{z} d}, \\
H^{(r e f 2)}=\frac{1}{2 D\left(k_{\perp}\right)}\left(1-\frac{k_{z} \varepsilon}{q_{z}}\right) e^{i q_{z} z_{r}+i k_{z} z_{l}}
\end{array}
$$

where $d=z_{r}-z_{l}$ is the plate thickness and

$$
D\left(k_{\perp}\right)=\cos \left(q_{z} d\right)-\frac{i}{2}\left(\frac{k_{z} \varepsilon}{q_{z}}+\frac{q_{z}}{k_{z} \varepsilon}\right) \sin \left(q_{z} d\right) .
$$

The zeroes of the function $D\left(k_{\perp}\right)$, whenever they exist, indicate the existence of nondecaying eigenmodes of the field in the plate. It is clear that $D\left(k_{\perp}\right)$ has no zeroes if the wavevector $k_{z}$ is real. This is why we saw no amplification in the preceding sections, where we only considered propagating waves.

For an arbitrary sign of $\varepsilon$, the equation $D\left(k_{\perp}\right)=0$ always has a solution if $q_{z}$ is real and $k_{z}$ is imaginary. These are the eigenmodes of a transparent plate placed into a nontransparent medium without dissipation. The nonpropagating field of the source is "amplified" near the values of resonant wavevectors $k_{\perp}^{(n)}$ corresponding to the eigenmodes of the plate.

But the most interesting result is obtained when both $k_{z}$ and $q_{z}$, are imaginary. In this case, the $D\left(k_{\perp}\right)$ can be written as 23 .

$$
D\left(k_{\perp}\right)=\frac{1}{2} e^{-\left|q_{z}\right| d}\left[1-\frac{1}{2}\left(\frac{\left|k_{z}\right| \varepsilon}{\left|q_{z}\right|}+\frac{\left|q_{z}\right|}{\left|k_{z}\right| \varepsilon}\right)\right]+\frac{1}{2} e^{\left|q_{z}\right| d}\left[1+\frac{1}{2}\left(\frac{\left|k_{z}\right| \varepsilon}{\left|q_{z}\right|}+\frac{\left|q_{z}\right|}{\left|k_{z}\right| \varepsilon}\right)\right] .
$$

To avoid cumbersome formulas, we analyze Eq. (17) for $\varepsilon \mu=1$. In this case,

$$
\left|k_{z}\right|=\left|q_{z}\right|=\sqrt{k_{\perp}^{2}-k_{0}^{2}}
$$

and the equation $D\left(k_{\perp}\right)=0$ becomes

$$
D\left(k_{\perp}\right) \equiv \frac{1}{2} e^{-\sqrt{k_{\perp}^{2}-k_{0}^{2}} d}\left[1-\frac{1}{2}\left(\varepsilon+\varepsilon^{-1}\right)\right]+\frac{1}{2} e^{\sqrt{k_{\perp}^{2}-k_{0}^{2}} d}\left[1+\frac{1}{2}\left(\varepsilon+\varepsilon^{-1}\right)\right]=0 .
$$

It is easy to see that Eq. (18) has a solution $k_{\perp}=k_{\perp}^{(r e s)}$ only at negative $\varepsilon$.

The larger the "amplification" of a nonpropagating wave, the closer $k_{\perp}$ is to its resonant value $k_{\perp}^{(r e s)}$. In other words, the smaller $D\left(k_{\perp}\right)$, the closer the wave to its resonance and, hence, the larger its amplitude at the output of the "cavity". As $\varepsilon \rightarrow-1$, the function $D\left(k_{\perp}\right)$ decays exponentially fast as $k_{\perp}$ approaches $k_{\perp}^{(\text {res })}$ 
from below and is exponentially large above the resonance point. Therefore, by convention, we can consider the domain $k_{0}<k_{\perp}<k_{\perp}^{(r e s)}$ to be close to the resonance and the domain $k_{\perp}>k_{\perp}^{(r e s)}$ to be far from the resonance.

From expressions (15) and (18), we obtain the field of the nonpropagating Fourier harmonic beyond the plane-parallel plate,

$$
\begin{aligned}
H^{(t r 2)}(z)=e^{\sqrt{k_{\perp}^{2}-k_{0}^{2}}(d-z)} & \left\{\frac{1}{2} e^{-\sqrt{k_{\perp}^{2}-k_{0}^{2}} d}\left[1-\frac{1}{2}\left(\varepsilon+\varepsilon^{-1}\right)\right]+\right. \\
& \left.\frac{1}{2} e^{\sqrt{k_{\perp}^{2}-k_{0}^{2}} d}\left[1+\frac{1}{2}\left(\varepsilon+\varepsilon^{-1}\right)\right]\right\}^{-1} .
\end{aligned}
$$

It follows from (19) that the field of the Fourier harmonic in the plane $z=2 d$ is close to unity, i.e., to its value in the source plane $z=0$, if the wavevector $k_{\perp}$ is in the "resonance" domain $k_{0}<k_{\perp}<k_{\perp}^{(r e s)}$. At the same time, it is exponentially small in the domain $k_{\perp}>k_{\perp}^{(\text {res })}$ (see Fig. 8] $)^{5}$. We see that in the plane $z=2 d$, the amplitudes and phases of the source field are the same as in the plane of the source, not only for propagating Fourier harmonics, but also for nonpropagating ones. In other words, both propagating and nonpropagating Fourier harmonics participate in the image formation. The size of the smallest details resolved in the image is given by the value $2 \pi / k_{\perp}^{(r e s)}$, which can be much smaller than the wavelength $\lambda=2 \pi / k_{0}$. As $\varepsilon \rightarrow-1, k_{\perp}^{(r e s)} \rightarrow \infty$, and we obtain a "perfect" lens.

In concluding this section, we note the following. In the resonance wavevector domain $k_{0}<k_{\perp}<k_{\perp}^{(r e s)}$, the first term in Eq. (18), the exponentially small one, is large compared to the second term. This is possible only if $\varepsilon$ differs from -1 by an exponentially small value. Assuming $\varepsilon=-1+\delta,|\delta| \ll 1$, we obtain from Eq. (18) that

$$
k_{\perp}^{(r e s)}=k_{0} \sqrt{1+\frac{\ln ^{2}(2 /|\delta|)}{k_{0}^{2} d^{2}} .}
$$

It follows from Eqn. (20) that for a given increase in the resolving power $k_{\perp}^{(\text {res })} / k_{0}$ of the lens, $\delta$ should decrease exponentially fast with the growing thickness $d$ of the lens:

$$
|\delta|=2 \exp \left\{-k_{0} d \sqrt{\left(\frac{k_{\perp}^{(r e s)}}{k_{0}}\right)^{2}-1}\right\} .
$$

\footnotetext{
${ }^{5}$ When this paper was in preparation, Ref. 24] appeared where the dependence shown in Fig. 8 was obtained from a numerical solution to the Maxwell equations. Because the case of a dissipative medium was considered in Ref. 24, the dependence there had a narrow maximum at $k_{\perp}=k_{\perp}^{(\text {res })}$ instead of a divergence, as in Fig. 8
} 


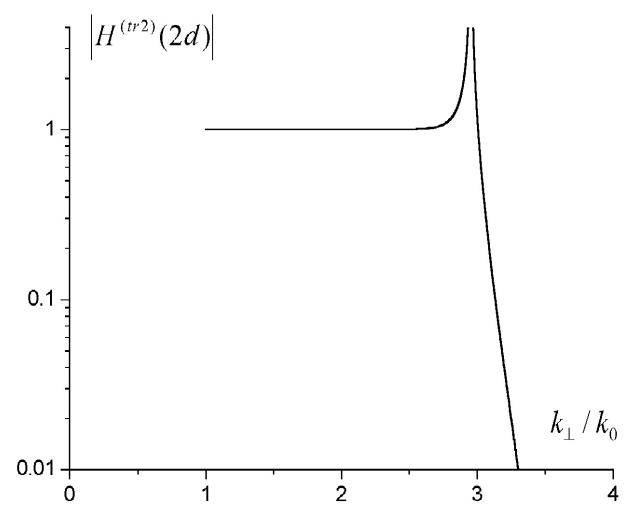

Figure 8: The amplitude of the non-propagating wave in the focal plane of a lens made of a left-handed material as a function of the wavevector [Eq.(19)]

For instance, for $k_{\perp}^{(r e s)} / k_{0}=3$ and $d=\lambda=2 \pi / k_{0}$ (this is the case for the dependence in Fig. 8),$\delta$ should be smaller than $10^{-7}$. Therefore, although a source can formally be imaged with any given precision, in practice only a small resolution increase is possible for a source placed at a small distance $(z \simeq \lambda)$ from the surface of a thin lens $(d \simeq \lambda)$. Such a combination of the object, lens, and image placed at a distance of the order of the wavelength or smaller should be considered not as a lens but rather as a complex source of radiation in a layered medium [25].

We make one more remark. For the cavity formed by the plate of a left-handed material to amplify the weak "input signal" (the nonpropagating wave from the source), this cavity should accumulate a large energy, which requires a long transient process. The larger the resolution that the lens is required to produce, the smaller the "input" level of the corresponding nonpropagating modes, and the larger the level of the field in the cavity and the time of the transient process. Most probably, ignoring this fact prevented the authors of Ref. [20] from observing the effect of a "perfect" lens when they performed numerical simulation of a time-limited wave packet propagating through a plane-parallel plate of a left-handed material.

\section{Conclusion}

In this work, we tried to give a review of left-handed media and some interesting effects related to them. Certainly, we could not mention most of the papers connected with this subject (see Fig. 10) but we hope to have mentioned the basic ones. 
Although the stream of papers is still huge (in 2003, several dozen papers have been published), the peak of the excitement has gone. In 1999 - 2002, almost every new statement was soon followed by arguments disproving it, but the present discussion is more productive. Now, very few researchers doubt the existence of left-handed media. Left-handed media have been realized in various ways by many experimentalists, and these works confirm both the unusual refraction law at the interface of two media and the focusing properties of a plane-parallel plate. The situation is somewhat worse with the perfect lens effect, because observation of this effect is possible only under very strict requirements to the parameters of the left-handed medium.

In this work, we did not touch upon subjects such as nonlinear optical effects at the interface of right-handed and left-handed media, one-dimensional periodic waveguide media containing left-handed elements, etc. All these ques- tions are related to the further development of this direction, while our aim was only to acquaint the reader with the basic principles of the physics of left-handed media.

This work was supported in part by the INTAS (grant No. 03-55-1921).

\section{References}

[1] Veselago V. G., Usp. Fiz. Nauk 92517 (1967) [Sov. Phys. Usp. 10, 509 (1968).]

[2] Veselago V. G. Fiz. Tverd. Tela 8, 3571 (1966). [Sov. Phys. Solid State 8, 2853 (1967).]

[3] Focus Issue: Negative refraction and Metamaterials Opt. Express 11 (7) (2003).

[4] Mandel'shtam L. I., Zh. Eksp. Teor. Fiz. 15, 475 (1945).

[5] Pendry J. B., Holden A. J., Stewart W. J., and Youngs I., Phys. Rev. Lett. 76, 4773 (1996).

[6] Pendry J. B., Holden A. J., Robbins D. J., and Stewart W. J., IEEE Trans. Microwave Theory Tech. 47, 2075 (1999).

[7] Silin R. A. Neobychnye Zakony Prelomleniya i Otrazheniya (Unusual Laws of Refraction and Reflection) (Moscow: Fazis, 1999).

[8] Silin R. A. and Chepurnykh I. P., Radiotekh. elektron 46, 1212 (2001) [J. Comm. Techol. Electron. 46, 1121 (2001).] 
[9] Silin R. A., Radiotekh. elektron 47, 186 (2002) [J. Comm. Techol. Electron. 47, 169 (2002).]

[10] Smith D. R., Padilla W. J., Vier D. C., Nemat-Nasser S. C., and Schultz S., Phys. Rev. Lett. 84, 4184 (2000).

[11] ShelbyR. A., Smith D. R., and Schultz S., Science 292, 77 (2001).

[12] Notomi M., Phys. Rev. B 62, 10969 (2000).

[13] Zhang Y., Fluegel B., and Mascarenhas A., Phys. Rev. Lett. 91, 157404 (2003).

[14] Bliokh Yu. P. and Felsteiner J., arXiv:physics/0311066.

[15] Pendry J. B., Phys. Rev. Lett. 853966 (2000).

[16] Valanju P. M., Walser R. M., and Valanju A. P., Phys. Rev. Lett. 88 187401 (2002).

[17] Pendry J. B. and Smith D. R., Phys. Rev. Lett. 90, 029703 (2003).

[18] Pacheco J., Jr., Gregorczyk T. M., Wu B.-I., Zhang Y., and Kong J. A., Phys. Rev. Lett. 89, 257401 (2002).

[19] Foteinopoulou S., Economou E. N., and Soukoulis C. M., Phys. Rev. Lett. 90, 107402 (2003).

[20] Loschialpo P.F., Smith D. L., Forester D. W., and Rachford F. J., Phys. Rev. E 67, 025602 (2003).

[21] Silin R. A., Opt. Spektrosk. 44, 189 (1978).

[22] Rupin R., J. Phys. Cond. Mat. 13, 1811 (2001).

[23] Smith D. R., Schurig D., Rosenbluth M., Schultz S., Ramakrishna S. A., and Pendry J.B., Appl. Phys. Lett. 82, 1506 (2003).

[24] Rao X. S. and Ong C. K., Phys. Rev. E 68, 067601 (2003).

[25] Veselago V. G., private communication. 